\title{
Hacia una sociedad más humana. El paradigma relacional de Pierpaolo Donati ${ }^{*}$
}

\author{
Towards a more humane society. \\ The relational paradigm of Pierpaolo Donati \\ Para uma sociedade mais humana. \\ O paradigma relacional de Pierpaolo Donati
}

Recibido el 23 de mayo de 2017. Aceptado el 29 de septiembre de 2017

Javier Ros Codoñer**

España

\section{Resumen}

Objetivos: comprender las características de

, Para citar este artículo:

Ros Codoñer, Javier (diciembre,

2017). Hacia una sociedad más

humana. El paradigma relacional

de Pierpaolo Donati. Ánfora,

24(43), 165-187. Universidad

Autónoma de Manizales. ISSN

0121-6538.

la sociedad de lo humano o sociedad dopo-moderna propuesta por Pierpaolo Donati como marco para la reintroducción del sujeto humano en los procesos sociales superando la dicotomía individuo/sistema. Metodología: estudio de Revisión que aplicó el análisis documental de fuentes y, secundariamente, recurrió a la entrevista en profundidad. Se accedió, además, a fuentes primarias para seleccionar y analizar la información relevante $y$, dada la magnitud de la obra del autor considerado, se hizo una revisión selectiva de libros y de artículos científicos. Resultados: el concepto de sociedad de lo humano es resultado de la fértil confrontación del paradigma relacional de Donati con el funcionalismo, especialmente el de Luhmann, así como de la respuesta a la modernidad reflexiva y a la posmodernidad. La activación de las nuevas relacionalidades sociales fruto de la creciente complejidad social conlleva la posibilidad

\footnotetext{
* Este artículo se deriva de la tesis doctoral "Al inizio è la relazione". La sociología relacional de Pierpaolo Donati defendida satisfactoriamente por Javier Ros Codoñer en la Universidad Católica de Valencia "San Vicente Mártir" en mayo de 2014. La investigación inicio en octubre de 2005 y culminó en mayo de 2014.

** Doctor en Sociología. Licenciado en Geografía e Historia. Profesor de la Universidad Católica de Valencia "San Vicente Mártir". España. Correo electrónico: javier.ros@ucv.es
} 
de distinguir lo humano de lo no humano en la sociedad y así facilitar la consecución de mayores cotas de humanidad frente a unas dinámicas sociales que parece excluirlas fruto de un modelo lib/lab de estructuración social. Conclusiones: la sociedad de lo humano no es una sociedad distinta de la existente, es resultado de poner en activo en ella la distinción humano/no humano de modo relacional y reflexivo con la consecuencia de hacer evidente y efectiva la posibilidad de prevalencia del sujeto humano frente al contexto o la estructura social.

Palabras clave: Sociología relacional; Humano; Individuo.

\section{Abstract}

Objective: to understand the characteristics of the humane society or dopo-modern society proposed by Pierpaolo Donati, as a framework for the reintroduction of the human subject into the social processes that overcomes the dichotomy of the individual and system. Methodology: a study review was applied to a documentary analysis of sources and an in-depth interview was conducted. Relevant information was selected and analyzed from the primary sources. A selective review of books and scientific articles was carried out due to the amount of published works from the author. Results: the concept of humane society is the result of the fruitful confrontation of the relational paradigm of Donati and the functionalism approach, especially that of Luhmann, as well as, the response to the reflexive concepts of modernity and postmodernity. The activation of the new social relationships results in increasing social complexity meaning the possibility of differentiating between the humane society from the non-humane society; thus, facilitating the achievement of greater levels of humanity against some social dynamics which seem to exclude them from a Lib-Lab model of social structure. Conclusions: the humane society is no different to that of the existing. It is the result of putting into practice the distinction between human and non-human, a relational and reflexive way of making evident and effective, the possibility of the prevalence of the human subject against the context or social structure.

Keywords: Relational sociology; Human; Individual.

\section{Resumo}

Objetivo: entender as características da sociedade do humano ou sociedade dopomoderna proposta por Pierpaolo Donati como um quadro para a reintrodução do 
sujeito humano nos processos sociais superando a dicotomia indivíduo / sistema. Metodologia: estudo de revisão que aplicou a análise documental de fontes e secundariamente, recorreu à entrevista em profundidade. Acedeu-se também a fontes primárias para selecionar e analisar informações relevantes e dada a magnitude da obra do autor considerado, se fez uma revisão seletiva de livros e artigos científicos. Resultados: o conceito de sociedade do humano é o resultado do paradigma relacional de Donati com o funcionalismo, especialmente aquele de Luhmann, assim como a resposta à modernidade reflexiva e pós-modernidade. A ativação das novas relacionalidades sociais, resultado da crescente complexidade social acarreta a possibilidade de distinguir o humano do não- humano na sociedade e assim, facilitar a obtenção de níveis mais elevados da humanidade contra algumas dinâmicas sociais que parece exclui-las, resultado de um modelo lib / lab de estruturação social. Conclusões: a sociedade do humano não é uma sociedade diferente da existente; É o resultado de ativar nela, a distinção humano / não humano de modo relacional e reflexivo, com a consequência de fazer clara e efetiva a possibilidade de prevalência do sujeito humano frente do contexto ou estrutura social.

Palavras-chave: Sociologia relacional; Humano; Individuo. 


\section{Introducción}

Muchos son los aspectos y áreas que la sociología actual aborda desde diversos paradigmas; sin embargo, ante la gran complejidad de la sociedad actual y la velocidad de cambio a la que se halla sometida es fundamental proporcionar un marco heurístico complexivo con el fin de poder incardinar en el mismo cualquiera de las parcialidades sociales que se aborden. A este desafío han respondido las ciencias sociales desde el primer momento de su existencia, puesto que la percepción del cambio social en el siglo XIX fue determinante para el alumbramiento de la sociología (Giner, 2008, pp. 625-626) y los paradigmas de comprensión y proposición social se sucedieron desde los primeros momentos.

Del recorrido por la historia de las ciencias sociales se puede concluir que dos han sido los grandes marcos conceptuales para el análisis: el marco de referencia sistémico u holista donde la sociedad, la estructura, posee sus dinámicas propias e impone desde fuera vínculos y coerciones a los individuos; y, en oposición al anterior, el marco de referencia individualista en el que la clave interpretativa arranca de los individuos y de sus acciones. En este segundo marco general, lo social carece de "realidad"; las realidades sociales son representaciones mentales que solo existen en los individuos.

Sin embargo, las propuestas paradigmáticas para conjugar ambos paradigmas -lo que se ha denominado como el problema del micro-macro link-no ha sido tarea sencilla y, como se verá a lo largo del artículo, autores tan significativos como Giddens (1984) o Coleman (1993) proporcionan un modelo interpretativo, pero que es incapaz de introducir adecuadamente al sujeto humano como verdadero agente de lo social. De este modo, es clave proporcionar una teoría sociológica que responda a esta situación y, al mismo tiempo, que sea capaz de ofrecer un análisis complexivo de la sociedad.

El paradigma relacional de Pierpaolo Donati, profesor de sociología de la Universidad de Bolonia, propone una respuesta, mediante el análisis y puesta en valor de la relación social, al secular conflicto entre individuo y sociedad, entre acción y estructura. Con ello, además, se consigue reintroducir lo humano en el campo de las ciencias sociales, tantas veces, y cada vez más, olvidado o negado.

Aquí reside la importancia de la sociedad de lo humano de Pierpaolo Donati: no sólo dota de centralidad al sujeto humano a través de su método relacional, sino que además consigue solucionar razonablemente el problema del micromacro link y dar una respuesta válida a las contradicciones inherentes a la fase histórico-social presente.

$\mathrm{Al}$ finalizar este trabajo, se pretende que el lector comprenda en qué consiste la sociedad de lo humano como propuesta de análisis y praxis alternativa a los 
distintos paradigmas sociológicos concretados en la posmodernidad o en la modernidad reflexiva. Así mismo, se busca presentar con claridad la posibilidad viable de reintroducir al sujeto humano en la vida social como protagonista y no sólo como parte y resultado de la estructura social.

Caracterizar la sociedad de lo humano o dopo-moderna y analizar someramente sus más básicas implicaciones es básico para entrar en una revisión competente de fenómenos sociales que desdibujan los contornos de lo humano como la medicina reproductiva, la investigación en células animales con fines terapéuticos en humanos, el ciborg, las relaciones con el mundo animal, etc. Es difícilmente alcanzable una sociedad cada vez más humana si no se lleva a cabo una conceptualización de lo humano que proporcione puntos de anclaje para la esfera normativa de la sociedad dado que, como se analizará más adelante, actualmente no existe una correlación necesaria entre lo social y lo humano.

\section{Metodología}

Este estudio aplicó la metodología cualitativa del análisis documental de y la entrevista en profundidad. Se accedió, principalmente, a fuentes primarias con el fin de detectar, seleccionar y analizar la información relevante para el objetivo fijado y, dada la magnitud de la obra del autor considerado, se hizo una revisión selectiva tanto de libros como de artículos científicos (Hernández Sampieri, Fernández Collado, Baptista, 2006, pp. 64-65). De modo complementario, se utilizó la entrevista en profundidad (Balcázar et al., 2013, pp. 57-91) para ajustar y clarificar algunos elementos teóricos desarrollados en la obra analizada.

El proceso se desarrolló en tres fases. En la primera, se recopiló la bibliografía de Pierpaolo Donati relacionada con el concepto trabajado a partir de fichas bibliográficas, hemerográficas y de información en formato electrónico. En la segunda fase, se seleccionaron autores relacionados con el particular con el objeto de profundizar en el análisis y la crítica de la teoría donatiana; igualmente se complementó la investigación con algunos fragmentos de una entrevista en profundidad realizada por el autor de este artículo al profesor Donati en 2008. Finalmente llevó a cabo la síntesis crítica y conclusiva de todo el material recopilado. 


\section{Resultados}

Los hallazgos de la investigación se exponen en tres momentos. En el primero, se exponen los análisis más importantes realizados sobre el particular en la sociología contemporánea, con énfasis en la perspectiva de la posmodernidad y el funcionalismo de Luhmann. En el segundo momento, se muestra la aportación de la sociología relacional de Pierpaolo Donati a la construcción del concepto de sociedad dopo moderna o sociedad de lo humano. Al final, se sintetizan los elementos fundamentales de la posibilidad de una sociedad de lo humano.

\section{Lo humano y el micro-macro link}

La propuesta de Pierpaolo Donati de la sociedad de lo humano o dopo-moderna ${ }^{1}$ se inicia con la descripción del mundo occidental contemporáneo y, como es habitual en su extensa obra, de la consiguiente crítica al funcionalismo (Donati, 1991, pp. 540-544; 2006c). El funcionalismo, en cualquier de sus modelos (Donati, 2006c) ha mostrado incapacidad de incorporar al análisis lo no funcional así como sus relaciones haciendo cada vez más difícil la introducción del elemento humano en dicho paradigma. Lo no funcional viene entendido en el funcionalismo como aquellos fenómenos sociales que ocurren al margen de la necesaria adaptación, según este paradigma, y que deben darse en toda sociedad de toda institución social. Así, realidades como la aceptación en la familia del individuo en fase terminal o del nasciturus con malformaciones se entienden como no funcionales y, por tanto, se hace del todo punto inviable su incorporación en el sistema teórico meramente adaptativo de cualquier tipo de funcionalismo.

El mundo contemporáneo genera la sensación de que la sociedad es cada vez más fragmentaria, arriesgada, incierta, anómica, fluctuante... No son pocos los análisis sociales que se enmarcan en este discurso descriptivo de lo social con ciertos rasgos pesimistas. Entre ellos cabe destacar el concepto de sociedad del riesgo del alemán Ulrich Beck (2006; 2008), la modernidad líquida del polaco Zygmunt Bauman (1999; 2015; 2017) o las caracterizaciones sociales del pensador alemán de origen coreano Byung-Chul Han (2012; 2013).

En todas las épocas se ha hablado de la deshumanización o decadencia de la sociedad; sin embargo, la gran diferencia respecto de situaciones anteriores

\footnotetext{
1. El uso de un término u otro es indistinto por parte del autor y así se va a seguir en el presente artículo. Se va a mantener el término italiano dopo-moderno dado que es el original y su traducción al castellano no sería lo necesariamente sintética que se requiere para este tipo de conceptos. En castellano se podría hablar de "la sociedad que está después de la moderna". Este concepto es central en el artículo y se detallará a lo largo del mismo.
} 
se encuentra, a juicio del italiano, en la emergencia dentro de lo social de lo no humano. En consecuencia, hay una paulatina escisión entre lo social y lo humano y cualquier realidad social y, por el hecho de serlo, ya no puede calificarse inmediatamente como humana. El origen de esta situación cabe buscarlo en la herencia que han dejado la modernidad y el propio funcionalismo con sus derivaciones (Donati, Colozzi, 2006, pp. 68-69). Herencia que se concreta en dos problemas:

a) Lo humano se ha reducido a lo social pues "todo contenido o hecho de la consciencia humana debe ser reconducida factores sociales determinantes (...) Es una visión ultrasocializada del hombre" (Donati, 1983, p. 59). Ello conduce a la pérdida del carácter trascendente de lo humano sobre lo social, dificultándose los posibles procesos de cómo lo humano puede generar nuevas formas sociales respecto a las dadas.

b) Se deriva de lo anterior el problema de cómo gestionar, si existen, los confines entre lo humano y lo no humano en lo social. Fenómeno éste que se evidencia por ejemplo en los debates éticos sobre el uso de células animales en terapias sobre humanos (Aznar, Gómez Tatay, 2017), en iniciativas como el Proyecto Gran Simio (Angulo, 2016) o en el debate entre transhumanistas y bioconservadores en el área de las disciplinas deportivas, por ejemplo (Sebastián, 2014, p. 118).

El contexto socio-histórico desde el que Donati parte para llevar a cabo su reflexión conducente a la sociedad de lo humano es la posmodernidad. A partir de los años setenta del pasado siglo surge la posmodernidad, que en el ámbito aquí tratado se caracteriza de forma ambivalente por dos elementos: por la disolución de la persona humana en la sociedad y por el continuo cambio entre los confines de lo humano y lo no humano en lo social. Para el pensamiento posmoderno, centrado en la subjetividad, no puede haber una respuesta cierta a las cuestiones sociales porque lo único cierto es que no puede haber certeza ni en los fundamentos ni en los fines (Rocca, 2011).

La posmodernidad solo constata un sistema adaptativo en consolidación, como bien propone el análisis funcionalista, que coloniza al ser humano a la vez que abre nuevas expectativas; de esta manera conduce a una sociedad diferente de la moderna. Claro ejemplo de este proceso es el desarrollo y difusión masiva de las tecnologías de la comunicación e información o las aportaciones constantes de la investigación biomédica. La sociedad se entiende como una gran máquina que se desarrolla para proteger al ser humano de los riesgos y del azar. No obstante, lo humano está fuera de lo social y se reduce a cuestiones de estética, de consumo, 
de formas de expresión más o menos irracionales (Lipovetsky, 2015). Se trata de una sociedad que vive el instante y se contrapone a lo social entendido como control racional y normativo (Lipovetsky, 1983).

Donati encuentra en la obra de Niklas Luhman el lugar donde reflexionar las cadencias argumentales del contesto posmoderno; de hecho, se puede afirmar que el paradigma relacional surge al hilo de la confrontación del italiano con el neofuncionalismo del alemán. Para Luhmann (1998) el sistema mecanicista social permite y empuja a los individuos a salir y volver a entrar en la sociedad. Esta presión de los mecanismos sociales sobre los individuos para comportarse autorreferencialmente, viene denominada en este autor como liberación de los individuos de la sociedad. La denominada liberación no ocurre en la sociedad sino fuera de ella, en el ambiente de la sociedad, en todo aquello que está fuera de las relaciones sociales concretas. Lo humano se ve como el impulso, el rumor externo respecto del sistema de comportamiento, de las reglas y mecanismos que llevan adelante la sociedad (Herrera, 2000, p. 186). Lo humano se identifica con las relaciones libres de condicionamiento. Las relaciones sociales organizadas son constrictivas, rutinarias, artificiales, abstractas y mecánicas. De este modo, lo social y lo humano van por vías diversas, confiándose a los sistemas técnicos la resolución de todo tipo de problemáticas.

En cuanto este entramado ambiental se hace más complejo, presiona a la sociedad para que gestione la propia complejidad del ambiente. En esta línea, una sociedad solo puede evolucionar si, al aumentar la complejidad externa, consigue aumentar su propia complejidad interna, mediante mecanismos de selección. Además, en el paradigma luhmanniano estos procesos son mecánicos, pertenecen a los propios ritmos evolutivos del sistema.

El neofuncionalismo, concretamente en Luhmann (1998), entiende que la sociedad está llamada a ser humana sólo bajo ciertos aspectos de la existencia, de tal manera que bajo apariencia de liberación del hombre realmente ofrece una solución opuesta. Para esta corriente, el hombre es producto de la sociedad y ésta puede ordenarlo como quiera siempre que esto sea compatible tanto con las elecciones admitidas por el estadio evolutivo alcanzado como por las posibilidades técnicas. Junto con esto, todos los procesos y elecciones derivados se califican como neutros, ya que no entran en juego las dimensiones que se consideran específicamente humanas. En esta misma línea el neofuncionalismo entiende que las patologías sociales no tienen sujeto humano; se trata de manifestaciones sistémicas. La sociedad contemporánea, siguiendo el paradigma neofuncional, dispensa a los sujetos de problemas de conciencia, de intencionalidades significativas, es decir, de la creación de sentido por los propios sujetos. Lo humano no se elimina sino que fluctúa a placer, libre de pensar o comportarse de manera irracional (Donati y Colozzi, 2006, p. 52). 
Junto con el paradigma luhmanniano, han sido varios los intentos por situar las relaciones entre lo humano y lo no humano en el ámbito de la sociología reciente. Autores tan destacados como Anthony Giddens han intentado revalidar lo humano como fuerza causal activa de las transformaciones sociales. Para el británico lo social tiene carácter humano como actualización de una característica sui generis de lo social-humano denominada agency, entendida como enlace (link) entre estructura y actor social; no obstante, para Giddens lo que hay de humano en lo social reside en la capacidad de los procesos colectivos para superar los vínculos situacionales y de la naturaleza, vista ésta siempre como límite (Giddens, 1984). Para Coleman (1993), autor destacado de la corriente constructivista del rational choice, lo humano en lo social es aquello que lleva a construir la sociedad según formas racionales; tras la desaparición de lo humano en las relaciones primarias a causa del tecnicismo y de la movilidad espacial, lo humano debe ser reconstruido a través de actores corporativos; a más racional una sociedad, más humana, tal y como sucede con la transformación del ambiente natural en ambiente artificial.

Donati detecta tres cuestiones no resueltas satisfactoriamente en estas aproximaciones (Donati, Colozzi, 2006, pp. 73-74):

a) En ellas no se evita el determinismo sociológico ya que es la sociedad quien marca las posibilidades de realización de lo humano.

b) En concomitancia con esto, se pierden los confines entre ambas realidades, lo humano es proyección de los estados interiores de los individuos o de las estructuras y modelos tanto culturales como subculturales del ser humano.

c) Finalmente, recogiendo la secular corriente utilitarista británica, la moralidad se traduce en utilidad de los individuos, bien entendida como socialización de los actores mediante una producción simbólica colectiva o simplemente como factor cultural de coerción externa.

El elemento clave a partir del cual Donati, a lo largo de su extensa obra, elabora la crítica de estos planteamientos sociológicos es el deficiente modelo explicativo a la hora de articular las conexiones entre el individuo y la estructura: no se detecta la relación social como elemento originario de lo social. De este modo, y de una manera u otra, no existe el equilibrio necesario entre lo micro y lo macro, por lo que consecuentemente acaba prevaleciendo una visión más o menos individualista, o una visión más o menos estructural-sistémica. Para superar dicha desconexión, el problema del micro-macro link, es necesario "el giro relacional", es decir, analizar lo social no desde la estructura o desde el individuo 
sino desde las relaciones, la clave reside en el "estar en relación” (Donati, 2015, p. 89).

\section{La sociedad dopo-moderna}

Como se ha visto, corrientes importantes del análisis social más o menos relevantes en la actualidad, junto con otras que no es posible presentar, abordan el problema la conexión entre estructura e individuo (micro-macro link) que hace prevalecer a la primera frente al segundo o viceversa. De un modo u otro, lo humano siempre queda minusvalorado, incluso eliminado, en aras del sistema/ estructura o de la autorrefencialidad desgajada de lo social.

Esta situación, junto con el propio análisis de los fenómenos sociales, es la que lleva a Donati a generar y articular, tanto en la vertiente analítica como en la vertiente práctica, su propuesta relacional donde se conjuguen adecuadamente estructura e individuo en función de poner en relevancia lo humano.

El paradigma relacional constata que la sociedad contemporánea se caracteriza por el progresivo distanciamiento entre lo humano y lo social. Lo social no es automáticamente humano: "La sociedad son tantas cosas juntas, que están juntas no porque el hombre las haya integrado, sino porque la vida social se desarrolla en unos umbrales de complejidad en los que deben coexistir muchas cosas diversas" (Donati, 1991, p. 512).

A lo largo de toda la obra de Pierpaolo Donati las referencia a lo humano y lo no humano no se llevan a cabo en sentido normativo, como juicio de valor, sino meramente descriptivo. Por no humano se entiende aquello que no es propiamente humano: el mundo vegetal y animal, las máquinas, mientras que cuando hace referencia a aquellas realidades, que aun proviniendo de agentes humanos niegan o distorsionan lo humano, se caracteriza como des-humano. En el caso de emitir un juicio ético éste solo puede ir referido al binomio humano/des-humano y nunca a lo no humano, dado que éste no admite juicio ético, solamente descriptivo (Donati, Colozzi, 2006, pp. 52,1).

Con el fin de dar un matiz mucho más humanista en la descripción, frente a términos como individuo o sujeto más propios de la sociología, Donati introduce en su análisis el concepto de persona (Donati, 2006b, p. 230). En ello se aprecia cierto sustrato filosófico en el autor italiano cuando describe que la situación mencionada expulsa a la persona como sujeto humano de los factores que explican las dinámicas sociales y culturales y, por tanto, cada vez resulta más difícil atribuir la cualidad de humano a lo social. Efectivamente, esta separación entre individuo y sistema se percibe en la sociedad occidental y en no pocas ocasiones el sistema se conduce autopoiéticamente; además, bajo una pretendida autorrealización cada 
vez mayor de los individuos, aparecen dinámicas de uniformización social. No obstante, con el paradigma relacional propuesto por Donati (Donati, 1983; 2015) queda suficientemente aclarada la relación entre individuo y sociedad, entendida ésta como trama relacional, lo que haría innecesario acudir a terminología filosófica, que puede conducir a equívocos.

El mencionado proceso de separación de lo social y lo humano se percibe como falta de sentido, como ausencia de metas (Bauman, 2005; 2010). Estos fenómenos llevan a dos consecuencias. En primer término se producen procesos de despersonalización y deshumanización en las relaciones, incluso llegándose a la confusión entre humano y no-humano (Donati, 1991, p. 475). Sin embargo, $\mathrm{y}$ al mismo tiempo, se genera la apertura a nuevas posibilidades relacionales, surgen situaciones sociales susceptibles de reconfigurar la relacionalidad tradicional en otra que pueda ser calificada de más humana. El análisis social no se debe dejar llevar por cierto pesimismo de carácter ideológico que deja de lado consecuencias no previstas en determinadas esquemas conceptuales. Junto con los primeros procesos mencionados, aquellos que llevan a la deshumanización social, no es menos cierto que una certera prospección social permite conocer nuevas relacionalidades humanizantes.

Dentro de estas nuevas posibilidades el profesor Donati encuadra el surgimiento de la sociedad dopo-moderna frente a la posmodernidad o la modernidad reflexiva con las siguientes palabras obtenidas a través de la entrevista en profundidad:

La sociedad relacional es la sociedad que tiene que producir, que tiene que generar lo humano de manera mediata, es decir, a través de una intención, de una relacionalidad propia, porque la sociedad no sigue siendo inmediatamente humana. Hasta hace unos años lo social era inmediatamente humano, era el producto de los hombres. Ahora lo social no es inmediatamente humano en el sentido que las comunicaciones, las tecnologías producen relaciones que no son inmediatamente humanas. En esta condición tenemos que buscar lo humano, una relacionalidad que es intersubjetiva entre sujetos no entre máquinas o artefactos. La sociedad de lo humano no es una cosa ideal, es la realidad de la sociedad que se genera de manera humana en el ambiente a través de una cierta relacionalidad. Muchas cosas no se producen de esta manera, sino de una manera no-humana, que no quiere decir inhumana. No humana significa no específica de la especie humana, del género humano; la sociedad de lo humano es la misma en la que vivimos, pero mirada desde el punto de vista de lo que hay de humano, dado que en lo social se dan elementos que no son humanos (Donati, 2008a). 
La modernidad reflexiva es entendida como la radicalización de la modernidad y como su expresión más madura (Beck, Giddens y Lash, 1999) y, la posmodernidad, como la crítica radical a la modernidad (Díaz, 2005). De ello, Donati deduce que sería adecuado hablar del fin de la modernidad, ya que ambas posturas conceptuales llegan a erosionar seriamente el proyecto moderno, hijo de la Ilustración (Donati, 2015). La sociedad dopo-moderna surge como respuesta relacional a la sociedad individualizada de la posmodernidad, donde muchas paradojas sociales proceden de la mencionada cada vez mayor distancia entre lo social y lo humano (Sebastián, 2014, p. 117).

La dopo-modernidad es presentada como la vía para salir de los dilemas de la modernidad, sea o no reflexiva, y de la postmodernidad. El concepto de sociedad dopo-moderna o sociedad de lo humano es la concreción conceptual de la sociedad emergente, fruto de una lógica relacional que supera la lógica funcionalista típica de las mencionadas sociedades (Sandoval y Garro-Gil, 2012). Si bien la sociedad humana "es la realidad social pensada y hecha por hombres como agentes de relaciones sociales, intersubjetivas y estructurales por tanto, inmediatamente dotadas de significado humano" (Donati, 2009, p. 70), la sociedad de lo humano o dopo-moderna pone "de manifiesto el surgir de nuevos códigos simbólicos inexistentes hasta el momento como consecuencia de los grandes procesos de industrialización, capitalización, globalización y desarrollo social iniciados durante la modernidad" (Donati, 2011, p. 4). En ella actores sociales actúan como productores-distribuidores-usuarios de los bienes relacionales, de modo que lo humano es intencionalmente buscado. Así se abre una vía para reintroducir al sujeto humano como protagonista del devenir social, objetivo éste primordial del paradigma relacional (Donati, 1983).

En la sociedad dopo-moderna la lógica relacional supera a la lógica de sesgo funcionalista típica de las sociedades modernas y posmodernas. Se trata de una concienciación de la necesidad de un nuevo modo de acción social ante la descomposición del Estado del bienestar (Herrera, 2001) y la necesidad humanizadora de los actores sociales y de los propios individuos (Lucio, 2007), muchas veces insatisfecha.

Para Donati, la sociedad dopo-moderna no piensa en el progreso lineal de la sociedad si no que entiende las formas sociales con una orientación hacia un proyecto que ni tiene porque ser evolucionista ni tampoco apertura total a la contingencia. La pieza clave se halla en que la sociedad se relaciona cada vez más. Junto con los valores modernos, que ahora se enfocan de otro modo (Donati, Colozzi, 2006, p. 81), han ido apareciendo nuevas realidades sociales fruto de los procesos de las últimas décadas en todos los ámbitos sociales y en todos sus niveles relacionales; desde la cotidianeidad y los fenómenos locales a los grandes flujos culturales, económicos y políticos globales. 
En las ciencias sociales, tanto los enfoques individualistas como los sistémicos están incapacitados para comprender y gestionar el mencionado proceso actual de diferenciación entre lo social y lo humano. Las dos grandes corrientes intervienen sobre los individuos o sobre las estructuras sociales; sin embargo; aquellos cada vez son más débiles y estas adquieren paulatinamente más autonomía mientras se descomponen. Ante esta situación, Donati entiende que las ciencias sociales deben saber aprehender lo que hay de humano en lo social, reconociendo así la capacidad que posee lo social para generar una sociedad de lo humano (Donati, 2009, p. 68).

\begin{tabular}{cc}
\hline $\begin{array}{c}\text { MODERNO } \\
\text { humano } \\
\text { humediatamente }\end{array}$ & $\begin{array}{c}\text { Do humano no procede directa- } \\
\text { mente de los social }\end{array}$ \\
\hline Progreso lineal e indefinido & Desarrollo sostenible y delimitado \\
\hline Lógica funcional & Lógica relacional \\
\hline $\begin{array}{c}\text { Explotación del entorno } \\
\text { entre Estado y sociedad civil }\end{array}$ & Ecología humana \\
\hline Estado nacional & Sociedad multicultural \\
\hline Constitucionalización política & $\begin{array}{c}\text { Constitucionalización de las esfe- } \\
\text { ras privadas (constitución civil) }\end{array}$ \\
\hline
\end{tabular}

Claves de lo moderno y lo dopo-moderno

Fuente: elaboración propia con base en Donati (2006b, p. 230)²

La distinción entre humano y no humano es relevante por una serie de motivos (Donati, 2007, pp. 84-85). Su pérdida comporta consecuencias inaceptables en el plano de la razón. Por una parte, lleva a la animación de realidades inanimadas, como los derechos de la Tierra, de las plantas... y a la atribución de subjetividad a los animales, como los derechos de los mismos. Por otra, comporta la atribución de realidad humana ipso facto a realidades no humanas, como la inteligencia artificial, los mundos virtuales... No obstante, la distinción se hace

2. Este gráfico procede de una obra de 2006 que ha sido superada en algunos aspectos; sin embargo, se usó en este estudio por la claridad que, en general, proporciona. De hecho Donati utiliza cada vez menos el término de sociedad global para hablar únicamente de sociedad dopo-moderna o sociedad de lo humano; de ahí que hayamos sustituido en la tabla aquel por éste. Igualmente, en 2006 utiliza el término multicultural siendo que posteriormente el autor italiano ha llevado una fuerte crítica sobre dicha conceptualización para utilizar el término de transculturalidad (Cfr.: Donati, 2008). 
imprescindible para la rehumanización de muchos ambientes de la vida social como pueden ser especialmente aquellos relacionados con las nuevas tecnologías de la comunicación e información.

La sociología relacional desarrolla un análisis en el que se busca no separar lo que es producto artificial de lo que, estando en ese ámbito, tiene raíces en la vida pre y metasocial. Lo que se cuestiona el profesor Donati ante las teorías de Luhmann (Donati, 2006c) es si se debe aceptar que sea la sociedad quien ordene al ser humano en su creciente indeterminación o que sea el individuo quien ponga orden en la sociedad. El modo en que la persona vive y construye la relación social puede clarificar la perspectiva relacional.

La novedad que describe Donati es la posibilidad de elaborar un paradigma dopo-moderno para las ciencias sociales que observe al individuo como sujeto relacional que crece y madura con y a través de las relaciones sociales, en el interior de una economía de la acción humana basada en los intereses últimos. Es decir, unos parámetros de acción social no eminentemente funcionales sino entretejidos de forma socialmente consciente con elementos trascedentes como pueden ser el altruismo, la solidaridad, el valor del débil o la creencia religiosa.

La modernidad se ha desequilibrado viendo sólo una hipersocialización, propia de la sociología y del neo-socialismo, modelo lab, o una hiposocialización, propia de la economía y del neo-liberalismo, modelo lib (Donati, 2002). El dilema que detecta Donati para la sociología relacional es comprender y explicar cómo el sujeto humano es una realidad tanto dependiente de la sociedad como dotada de autonomía. Siguiendo a Archer (Archer, 1995; 2003), Donati afirma que con la epistemología realista crítica relacional (Donati, 1991, pp. 315-322) se pueden comprender algunas operaciones que de otro modo resultan inabarcables (Donati, 1983, pp. 110-114) como por ejemplo:

- Ver la realidad de la persona humana no como reconducible a producto social o concepto ideal

- Ver la identidad del self y su capacidad de madurar en las interacciones sociales y a través de ellas

- Ver a la persona como sujeto singular y único que habita cuatro órdenes de la realidad (natural, práctico, social y espiritual) que debe atravesar para humanizarse.

Esta elaboración teórica se aprecia claramente en el análisis del proceso de socialización, como lugar de conexión concreta entre sociedad e individuo, micro-macro link. La socialización no se enfoca en clave determinista como un proceso impositivo sobre el individuo donde éste no tiene nada que aportar sino que viene entendida como proceso que se actualiza a través de una conversación 
interior (Donati, 2006a). En esta conversación interior el individuo elabora su autoconocimiento por lo que la socialización deviene en un proceso activo del sujeto en el interior de condicionamientos externos.

Con este enfoque el individuo recupera su prioridad lógica y temporal en confrontación con los procesos sociales frente a las teorías de la modernidad que entienden la socialización como una mera adaptación del sujeto a modelos culturales prevalentes en su contexto socio-cultural. La relación entre identidad personal e identidad social se entiende como fenómeno emergente, ambas identidades interactúan con la prevalencia de la primera (Donati, Colozzi, 2006, pp. 98.108). La evidencia es que el ser humano no puede dejar de referir a sí mismo el sentido de las relaciones sociales a través de la conversación interior por lo que ciertamente origina redes sociales y vive en ellas, pero no se diluye en su seno.

\section{La búsqueda social de lo humano en la sociedad dopo-moderna}

Como ya se ha afirmado, actualmente la sociedad no puede ser considerada inmediatamente como humana, desde el momento en que lo social resulta de la interacción de elementos humanos y no humanos, que la modernidad ha hecho crecer como realidades autónomas aunque interpenetradas. En este contexto de crecimiento y desarrollo complejo de las mediaciones de todo tipo, es necesario perseguir lo humano explícitamente

En una situación donde las relaciones sociales no son humanas per se (Donati, 2009, p. 70), se ha de proceder constantemente a distinguir aquello que podemos decir sociedad de lo humano, siendo ésta aquella cuyas relaciones están producidas por sujetos que se orientan recíprocamente a partir de un sentido suprafuncional y que es capaz de generar bienes relacionales (Donati, 1994, p. 171; 1995).

La sociedad de lo humano es distinta de otros tipos de sociedad cuyas relaciones sociales no necesariamente se corresponden con requisitos propiamente humanos (Donati, 1994, p. 170; 2006d). A lo largo de sus escritos, el profesor italiano no entiende la sociedad de lo humano como otra sociedad sino como un modo de generar y estar en las relaciones para ir más allá del puro mecanicismo social. Este tipo de sociedad se interroga constantemente por lo humano, ya que no se da inmediatamente en el ambiente.

Al respecto, se propone un cuadro conceptual explicitando la perspectiva relacional en este particular (Donati, Colozzi, 2006, p. 89): 
- Lo social es humano en tanto en cuanto es relacional

- Las características de lo humano (los elementos biológicos, afectivos, cognitivos y simbólicos) están relacionados entre sí; la relación es el contexto de los elementos singulares, y las relaciones son colocadas de nuevo en nuevos contextos relacional.

- La acción social es humana en tanto que no presenta solo uno de los elementos, sino como forma específica o prevalente

- Entre lo humano y lo social hay una distancia pero también interdependencia e interacción, por tanto historia

- La calidad humana de las formas sociales reside en dos elementos fundamentales. El primero es la particular gestión de los puntos de contacto entre relaciones sociales, intersubjetivas y estructurales, y la latencia social; con ellos se está haciendo referencia a la conexión con los valores y, por tanto, con los fines trascendentes. El segundo es la consecución de una relacionalidad que distinga inmanencia y trascendencia de aquello que en lo humano no es reducible a lo social; se trata de huir del sociologismo para introducir en el análisis aquello que en el individuo y sus relaciones no puede ser reducido a socio-cultural.

Donati (2007) afirma que "la sociedad occidental moderna al liberar al máximo las fuerzas que exaltan la potencialidad de las relaciones sociales debe afrontar el desorden que se sigue de los procesos que se derivan de la continua diferenciación y fluctuación de las mismas relaciones que han generado" (pp.105-106). Actualmente las dinámicas sociales vienen marcadas por la continua destrucción y creación de relaciones sociales. Este proceso genera en la sociedad la necesidad de mantener las conexiones significativas entre la cualidad propiamente humana de las relaciones sociales y los determinismos que de ellas puedan surgir. Al mismo tiempo, ha de saber crear y gestionar las relaciones sociales de manera que se reduzcan los posibles efectos perversos y patologías. Entendida la patología social como ruptura, eliminación o distorsión de las relaciones, bien por claro individualismo bien por la emergencia de sistemas sociales que incapacitan la generación de sentido en las relaciones interhumanas, pues las constriñen y formalizan dentro de estructuras que no permiten corresponder a las exigencias vitales de los agentes sociales (Donati, 2007, p. 107).

Dado que dichas patologías surgen cuando los sujetos quedan atrapados en los mecanismos de las redes sociales ${ }^{3}$, se hace necesaria una teoría y una praxis

3. No se está haciendo referencia al concepto actual, y mayoritariamente difundido, de redes sociales como el tejido virtual en el que cada vez se mueve más el individuo mediante mecanismos como Facebook, Twitter, Instagram, Whatsapp... El concepto de red social viene claramente referido al tejido relacional que siempre se da en la sociedad, tanto entre individuos como entre instituciones o entre unos y otros. 
que, gestionando las propias redes sociales, pueda liberar a los sujetos humanos de las tramas de dichas redes, sin destruirlas ni negarlas, sino a través de itinerarios de distanciamiento, modificación y morfogénesis que paulatinamente vayan reintroduciendo lo humano. En el caso de las redes sociales virtuales este análisis lo lleva a cabo muy acertadamente Byung-Chul Han en su obra En el enjambre (2014). Para este autor "la comunicación digital hace que se erosione fuertemente la comunidad, el nosotros. Destruye el espacio público y agudiza el aislamiento del hombre” (p. 75); de afirmaciones como ésta concluye la necesidad de un replanteamiento social que, en cierto modo sigue en paralelo el mismo planteamiento acerca de la reintroducción de lo humano de Pierpaolo Donati.

\section{Conclusiones}

Pierpaolo Donati aporta al análisis de la sociedad actual desde un planteamiento globalizador de la misma, la sociedad de lo humano o dopo-moderna. Se trata de una propuesta que hace frente a las descripciones sociales más difundidas actualmente, como son la de la posmodernidad o la de la modernidad reflexiva o avanzada, haciendo especial hincapié en la reintroducción del sujeto humano como protagonista frente a la omnipresencia de la estructura. Junto con ello, se consigue dar una respuesta, a través del análisis de la relación social, al problema de cómo conectar individuo y sociedad, acción y estructura (micromacro link). De hecho, la sociología relacional entiende que la acentuación de lo humano en el análisis social es la única vía para llevar a cabo satisfactoriamente dicha conexión.

El planteamiento relacional que conduce a la propuesta de la sociedad de lo humano no supone el rechazo a lo contingente o a la complejidad del mundo actual, como ha demostrado sobradamente la extensa bibliografía de Pierpaolo Donati y la obra de autores como Ivo Colozzi (2009), Paolo Terenzi (2016), Sergio Bellardinelli (2015), Ricardo Prandini (2010), Andrea Maccarini (2003) o Pablo García Ruíz (2009).

Siguiendo a Herrera (2000, pp. 187-188) en esta cuestión dos son los aciertos decisivos de la aportación de Donati. Por una parte permite redefinir lo social más allá de ser una proyección individual o algo independiente del sujeto; por otra parte, hace posible una teoría “crítica” con la "modernización” centrada en algunos de sus límites humanos sin caer en la eliminación de la contingencia, sino que ésta viene redefinida constantemente por relacionamientos posteriores.

El análisis relacional busca afrontar la complejidad social y la presencia de lo contingente en ella teniendo presente la indeterminación entre los límites de lo humano y lo no humano que se dan en las sociedades avanzadas. "Toda forma social 
expresa una determinada calidad en las relaciones y ésta es la que decide el tipo y grado de humanidad que contiene esa forma social respecto de otras" (Donati, 1994, p. 172). Con ello, la visión propia de la sociología relacional es la de una sociedad subsidiaria de la persona humana donde las relaciones sociales pueden favorecer o dificultar el desarrollo de lo humano. Las formas sociales tienen su propia realidad, propiedades, mecanismos y poderes emergentes que deben ser constantemente humanizados nada más se produce el efecto emergente. Esta es la propuesta de la sociedad dopo-moderna (Donati, Colozzi, 2006, p. 48).

Con todo, los rasgos definitorios de la sociedad de lo humano podrían concretarse en los siguientes:

- Surge como propuesta analítica y práctica a los paradigmas sociológicos herederos directos, por asunción o rechazo, de la modernidad

- Busca dar una respuesta propositiva en la búsqueda de lo humano en el marco global de la paulatina separación entre lo social y lo humano en la sociedad actual

- Reintroduce al sujeto humano como protagonista del devenir social a través de la reconfiguración relacional, donde esta lógica relacional supera la lógica funcionalista

- Permite la distinción entre lo humano y lo no humano facilitando una relacionalidad y normatividad no fruto de los mecanismos y estructuras (sistema político, económico...) sino de la reflexividad tanto de los actores sociales como de los individuos

- Da una respuesta adecuada al problema de la conexión entre individuo y sistema (micro-macro link) frente a otras propuestas que devienen normalmente en uno de los dos extremos.

La dopo-modernidad o sociedad de lo humano no significa el fin de la historia o del progreso, sino el fin de los mitos de la modernidad, que son reacios a morir adoptando constantemente nuevas formas y escenarios. La sociedad dopo-moderna no es vista por Donati como una imposición de corte tecnócrata o autoritario, sino como una puesta en valor del sujeto humano en todos sus ámbitos de actuación de un modo eminentemente relacional frente a una modernidad avanzada que pretende implementar mayor fuerza si cabe a la estructura social heredada de la modernidad. En última instancia la diferencia fundamental entre el funcionalismo, en cualquiera de sus concreciones, y la sociología relacional es que tanto los hechos como las relaciones dependen esencialmente del sujeto humano y no del contexto o estructura social. 


\section{Referencias}

Angulo, M. (2016). Las fronteras de la persona. El valor de los animales, la dignidad de los humanos. Xihmai, 131-138.

Archer, M. (1995). Realist Social Theory: the Morphogenetic Approach. Cambridge: Cambridge University Press.

Archer, M. (2003). Structure, Agency and the Internal Conversation. Cambridge: Cambridge University Press.

Aznar, J., Gómez Tatay, L. (6 de febrero de 2017). Observatorio de bioética. Universidad Católica de Valencia. Obtenido de www.observatoriobioetica. org: http://www.observatoriobioetica.org/2017/o2/organos-humanosavances-en-la-produccion-quimeras-humano-animales/18160

Balcázar, P., González-Arratia, N.I., Gurrola, G.M., Moysén, A. (2013). Investigación cualitativa. México: Universidad Autónoma del Estado de México.

Bauman, Z. (1999). Modernidad líquida. Buenos Aires: Fondo de Cultura Económica.

Bauman, Z. (2005). Identidad. Oviedo: Losada.

Bauman, Z. (2010). Vida líquida. Barcelona: Paidós.

Bauman, Z. (2015). Ceguera moral. La pérdida de sensibilidad en la modernidad líquida. Barcelona: Paidós.

Bauman, Z. (2017). Retrotopía. Barcelona: Paidós.

Beck, U. (2006). La sociedad del riesgo global. Madrid: Siglo XXI.

Beck, U. (2008). La sociedad del riesgo mundial: en busca de la seguridad perdida. Barcelona: Paidós.

Beck., U., Giddens, A., Lash, S. (1999). Modernización reflexiva: política, tradición y estética en el orden social moderno. Madrid: Alianza Editorial. 
Belardinelli, S. (2015). La sfida dell'educazione oggi. En S. Bellardinelli, L'urgenza di un nuovo umanesimo (págs. 103-121). Venecia: Orthotes Editrice.

Coleman, J. (1993). The Rational Reconstruction of Society. American Sociological Review, 1-15.

Colozzi, I. (2009). Sociologia delle istituzioni. Nápoles: Liguori.

Díaz, E. (2005). Posmodernidad. Buenos Aires: Biblos.

Donati, P. (1983). Introduzzione alla sociologia relazionale. Milano: Angeli.

Donati, P. (1991). Teoria relazionale della società. Milano: Angeli.

Donati, P. (1994). Sulla distinzione Umano/Non Umano. Per una sociologia del duemila. Il Mondo, 158-177.

Donati, P. (1995). Tradizione sociologica e sfida post-moderna: ripartire dalla distinzione unamno/non umano? En R. Gubert, \& L. Tomasi, Teoria sociologica ed nvestigazione empirica (págs. 237-271). Milano: Angeli.

Donati, P. (1999). La ciudadanía societaria. Granada, España: Universidad de Granada.

Donati, P. (2002). Ciudadanía y sociedad civil: dos paradigmas (ciudadanía lib/ lab y ciudadanía societaria). REIS, 37-64.

Donati, P. (2006a). La conversazione interiore: un nuovo paradigma (personalizzante) della socializzazione. En M. Archer, La conversazione interiore. Come nasce l'agire sociale (págs. 9-42). Trento: Erickson.

Donati, P. (2006b). Hacer sociología en la època de la globalización. En P. Donati, Repensar la sociedad. El enfoque relacional (págs. 205-246). Madrid: Ediciones Internacionales Universitarias.

Donati, P. (2006c). La comprensión del cambio social. En P. Donati, Repensar la sociedad. El enfoque relacional (págs. 165-204). Madrid: Ediciones Internacionales Universitarias. 
Donati, P. (2006d). Che cosa significa leggere la società? Una sociologia per il XXI secolo. En P. Donati, Una introduzione allo studio della società (págs. IX-XVII). Padova: Cedam.

Donati, P. (2007). La sociedad es relación. En P. Donati, Repensar la sociedad. El enfoque relacional. (págs. 49-164). Madrid: Ediciones Internacionales Universitarias.

Donati, P. (2008a). Cuestiones de sociología relacional. Pamplona: entrevista realizada por J. Ros el 19 de noviembre.

Donati, P. (2008b). Oltre il multiculturalismo. La ragione relazionales per un mondo comune. Bari: Laterza.

Donati, P. (2009). La società dell'umano. Genova-Milano: Marietti.

Donati, P. (2011). Relational Sociology: a ew Paradigm for the Social Sciences. London: Routledge.

Donati, P. (2015). El reto educativo: análisis y propuestas. Educación y educadores, 307-329. Recuperado de Educación y educadores: http:// educacionyeducadores.unisabana.edu.co/index.php/eye/article/ view/5721/3977

Donati, P. (2015). Manifesto for a Critical Realist Relational Sociology. International Review of Sociology, 1, 86-109.

Donati, P., Colozzi, I. (2006). Il paradigma relazionale nelle scienze sociali: le prospettive sociologiche. Bolonia: Il Mulino.

García Ruíz, P. (2009). Repensar el consumo. Madrid: Eiunsa.

Giddens, A. (1984). La constitución de la sociedad: bases para la teoría de la estructuración. Buenos Aires: Amorrortu.

Giner, S. (2008). Historia del pensamiento social. Barcelona: Ariel.

Han, B.-C. (2012). La sociedad del cansancio. Barcelona: Herder.

Han, B.-C. (2013). La sociedad de la trasparencia. Barcelona: Herder. 
Han, B.-C. (2014). En el enjambre. Barcelona: Herder.

Hernández Sampieri, R., Fernández Collado, C., Baptista, P. (2006). Metodología de la investigación. México: Mac Graw Hill.

Herrera, M. (2000). Representaciones de la sociedad: de la modernidad a la posmodernidad. Papers, 61, 163-190.

Herrera, M. (2001). Las políticas sociales en el Welfare Mix. REIS, 71-93.

Lipovetsky, G. (1983). La era del vacío. Barcelona: Anagrama.

Lipovetsky, G. (2015). La estetización del mundo. Barcelona: Anagrama.

Lucio, N. (2007). La società dopo-moderna. Dal rischio all'emergenza. Perugia: Morlacchi.

Luhmann, N. (1998). Sistemas sociales; líneas para una teoría general. Barcelona: Anthropos.

Lyotard, J.-F. (1987). La condición posmoderna. Informe sobre el saber. Madrid: Cátedra.

Maccarini, A. (2003). Lezioni di sociologia dell'educazione. Padua: Cedam.

Prandini, R. (2010). Soggettività sociali riflessive. La costituzione di un "noi" riflessivo. Sociologia e Politiche Sociali, 79-111.

Proyecto Gran Simio. (29 de abril de 2017). Recuperaado de http:/ / proyectogransimio.org/.

Rocca, A. (2011). La Posmodernidad. Nuevo régimen de verdad, violencia metafísica y fin de los metarrelatos. Nómadas, 29(1), 1-16.

Sandoval, L.Y., Garro-Gil, N. (2012). La sociología relacional: una propuesta de fundamentación sociológica para la institución educativa. Educación y educadores, 247-262. 
Sebastián, F. (2014). Sociologia, etica e commercializzazione dello sport: il contributo della porspettiva relazionale di Pierpaolo Donati. Sociologia e Politiche Sociali, 17, 115-133.

Terenzi, P. (2016). Lessico della sociologia relazionale. Bolonia: Il Mulino. 